\title{
Enhanced Corrosion Resistance of Silicone-Coated Stents by Plasma Treatment
}

\author{
H.B. KIM ${ }^{a}$, K.Y. BAIK ${ }^{b}$, M.H. MoON ${ }^{a}$ AND C.K. SUnG ${ }^{a, *}$ \\ ${ }^{a}$ Department of Radiology, Seoul National University College of Medicine, SNU-SMG Boramae Medical Center, \\ 20, Boramae-ro 5-gil, Dongjak-gu, Seoul, 156-707, Republic of Korea \\ ${ }^{b}$ Plasma Bioscience Research Center, Kwangwoon University, \\ 20 Kwangwoon-gil, Nowon-Gu, Seoul139-701, Republic of Korea
}

\begin{abstract}
The interfacial bonding of silicone-coated stents was characterized by field emission scanning electron microscopy and anodic potentiodynamic polarization tests. The coaxial in-lens secondary electron imaging revealed partial bonding between silicon rubber and nitinol alloy strips in both primer-treatment and plasma-treatment cases. Several strands between the polymer and stent substrate were observed in both cases and it seems that the bonding strength is the same. However, potentiodynamic polarization tests have shown that the corrosion rates of the plasma-treated nitinol stents were less than half of that of the primer-treated stents, indicating higher corrosion resistance. This enhanced corrosion resistance may be attributed to the modification of the physical properties of the surface of the stents by plasma treatments, making the oxide layer more effective than that of the primer-treated stents.
\end{abstract}

DOI: 10.12693/APhysPolA.129.857

PACS/topics: 81.65.-b

\section{Introduction}

Nitinol (NiTi) is an alloy of nickel and titanium which has been used in the construction of medical devices including self-expanding stents to brace the inside circumference of a tubular passage, such as cardiac, peripheral vascular, biliary, and gastrointestinal. It is now well understood that nitinol requires controlled processing to achieve optimal shape memory and superelastic properties $[1,2]$. In the same way, surface processing is required in order to promote optimal corrosion resistance and biocompatibility [2]. Oxide layers could give these roles to medical stents [3]. In vivo studies of the nitinol alloy material have shown that corrosion of the protective titanium (Ti) oxide layer occurs under certain conditions, resulting in the release of deleterious nickel $(\mathrm{Ni})$ ions $[4,5]$, which can provoke side effects. Non-biodegradable polymer coatings may provide a solution for this problem, as they can serve as a shield against corrosion and also as a platform for improving the biocompatibility of devices $[6,7]$. Silicone rubber is considered as one of the most biocompatible biomaterials and has been used in diverse biomedical applications. Coating with silicone is known to make stents being able to withstand high strains during stent crimping and development without peeling or fracture, and maintain integrity for extended periods in vivo [8].

Since the breakdown of the coating layer causes a release of irritant or toxic ions from the metallic stent, there have been many trials to keep bond strength. The bond

*corresponding author; e-mail: sckmd@hanmail.net strength of the coating is highly dependent on the substrate surface conditions [9], and a variety of treatments can be used to modify the surface and augment the bond strength. One approach is to increase the area available for bonding by increasing the surface roughness, but this does not provide the desired efficacy for a long time. Thus, surface chemical modification with primer has been used commonly to enhance chemical bond strength with silicon. On the other hand, plasma treatment has been used to modify the physical properties of the surface of stents and make the oxide layer more uniform for better durability [10-14]. Here, we report the comparison between the chemical and physical surface treatments by using field emission scanning electron microscopy and anodic potentiodynamic polarization tests.

\section{Materials and methods}

\subsection{Materials and stents}

Medical grade $\mathrm{NiTi}$ alloy strips $\left(\begin{array}{llll}43.3 & \% & \mathrm{Ti}\end{array}\right.$ and $56.6 \% \mathrm{Ni}$ ) of $0.08 \mathrm{~mm}$ diameter with the original oxide layer were manufactured by NDC Corp (Fremont, CA, USA). They were formed into the shape of a meshed metallic tube following the production process of M.I.Tech Corp, Ltd (Pyeongtaek-Si, Gyeonggi-Do, Korea). These stents were thermally treated at $530^{\circ} \mathrm{C}$ in a salt bath, accompanying oxidation on the surface after being cleaned in ultrasonic baths of alcohol and then deionized water. For corrosion tests, straightened wires were separately made $(1.0 \mathrm{~mm}$ in diameter and $100 \mathrm{~mm}$ in length) and subjected to the same process of thermal treatment.

\subsection{Plasma surface modification}

To modify the physical properties of the surface of the $\mathrm{NiTi}$ strips that were thermally treated and oxidized, 
a plasma etching system from Geniatech Corp., Ltd. (Suwon-Si, Gyeonggi-Do, Korea) was employed with a high-frequency electric field of $13.56 \mathrm{MHz}$. The plasma etching was carried out for all samples, including the straightened wires for corrosion tests, under the pressure of 16 mTorr at room temperature, at different applied voltages, flow rates of oxygen and argon gases, and treatment times (Table I).

\section{TABLE I}

Plasma processing conditions for treating the stents and the NiTi wires.

\begin{tabular}{c|c|c|c|c|c|c}
\hline \hline Items & $\mathrm{A}$ & $\mathrm{B}$ & $\mathrm{C}$ & $\mathrm{D}$ & $\mathrm{E}$ & $\mathrm{F}$ \\
\hline $\begin{array}{c}\mathrm{Ar} \\
{[\mathrm{cc} / \mathrm{sec}]}\end{array}$ & 20 & 20 & 20 & 20 & 20 & 20 \\
$\begin{array}{c}\mathrm{O}_{2} \\
{[\mathrm{cc} / \mathrm{sec}]}\end{array}$ & 15 & 15 & 15 & 15 & 15 & 30 \\
$\begin{array}{c}\text { Voltage } \\
{[\mathrm{kV}]}\end{array}$ & 1.2 & 1.2 & 1.25 & 1.25 & 1.3 & 1.3 \\
$\begin{array}{c}\text { Time } \\
{[\mathrm{min}]}\end{array}$ & 3 & 10 & 5 & 10 & 5 & 5
\end{tabular}

\subsection{Coating of NiTi with silicone}

NiTi strips modified by the plasma etching were subjected to liquid silicone rubber coating (Nusil Technology, Carpinteria, CA, USA) just after plasma treatment and then thermally treated at $150^{\circ}$ for 24 hours to evaporate the solvent. The controls that were first treated with the specially formulated primer (Nusil Technology), were coated with silicone at $50^{\circ} \mathrm{C}$ for 30 minutes according to the manufacturer's instructions, and then dried. The wires for corrosion tests were also coated with the silicone and thermally treated as in the case of stents. In case of the control wire, these processes were applied after adhering the primer to the wires.

\subsection{Field emission scanning electron microscopy} (FESEM)

Samples were fractured to expose cross-sections using very sharp scissors. The fractured samples were mounted on a metal stub and sputter-coated ( $c a .10 \mathrm{~nm}$ thick) with platinum using a coater (SCD 005, BAL-TEC, Balzers, Liechtenstein). They were observed with a field emission scanning electron microscope (Supra 55VP, Carl Zeiss, Oberkochen, Germany) at $2 \mathrm{kV}$. Secondary electrons were collected using a coaxial in-lens annular type detector.

\subsection{Potentiodynamic behavior}

To determine the corrosion potential and the corrosion rates of the samples, an anodic potentiodynamic polarization test was employed. An electrochemical cell was constructed, consisting of a saturated calomel reference electrode (SCE), pure Pt counter electrode, and a working electrode. The working electrode was dipped into de-aerated $0.9 \%$ sodium chloride solution, $50 \mathrm{~mm}$ from the electrode end. The electrolyte solution was used as a simulant of human body fluid and consisted of $138 \mathrm{mEq} / \mathrm{l} \mathrm{Na}{ }^{+}, 106 \mathrm{mEq} / \mathrm{l} \mathrm{Cl}^{-}, 4.4 \mathrm{mEq} / 1 \mathrm{~K}^{+}$extracellularly [15]. The electrolyte was assumed to be a homogeneous medium, ignoring local variations due to flowing interstitial fluids in human tissues. Before the polarization test, the working electrode was allowed to stabilize in the electrolyte solution for 10 minutes to reach an equilibrium state with no net current flow to the surface of the metal. The potentiodynamic polarization test was carried out at a scan rate of $5 \mathrm{mV} / \mathrm{s}$ using a computer-controlled 263A Potentiostat/Galvanostat (Princeton Applied Research, Oak Ridge, TN, USA) [16].

\section{Results and discussion}

In order to investigate the bonding strength between NiTi strips and silicon polymer, we have observed the interface between them. Figure 1 shows the surface of a stent, the cross-sectional views of the NiTi strips, and the linked and unlinked parts between the polymer and the NiTi strip of the primer-treated NiTi sample (Con.) and of the plasma treated NiTi samples $(\mathrm{A}-\mathrm{F})$. The A-F samples had various plasma treatment conditions including different applied voltages, flow rates of oxygen and argon gases, and treatment times, as shown in Table I. In the cross-sectional views, it was found that the silicone coating on the surface of NiTi alloy has kept an almost uniform thickness of over $10 \mu \mathrm{m}$. In contrast to the expectations, the interfaces consisted of the linked parts as a mechanical bond and the unlinked parts, regardless of the treatment methods. These images indicate the bonding was not strong but partially formed in the interface even with the primer treatment and the plasma treatment. Here it should be pointed out that we could not avoid mechanical damages in the interface with our cutting method by using scissors. Even when we tried liquid nitrogen to harden our sample, the gap was not reduced due to the severe shrinkage of metal in low temperature. In order to observe innate interfaces, more delicate sample preparation should be applied. However, we can say that the suppressing strength by the scissors was similar in all samples, because we applied same method to all of them.

Even though the bond between the NiTi and silicone polymer was not tight, the innate $\mathrm{TiO}_{x}$ layer and the silicon coverage should protect the $\mathrm{Ni}$ ion release from the stent into the human body. The production of a protective Ti-rich oxide layer on the surface of NiTi alloys used in medical applications also improves the corrosion resistance of the material in physiological conditions [17-19]. However as shown in Fig. 1, the silicon-detached surface of NiTi is not smooth, which can be due to the remained silicon or the innate oxide layer surface. In order to clarify it, we have observed both sides, the surface of silicone and the NiTi surface under higher magnification (Fig. 2). The surface of silicon shows many long cleavages and large peeling areas (Fig. 2a), and the metal surface shows the oxide with cracks (Fig. 2b). This is why the stent even with the silicone coverage shows toxicity when 


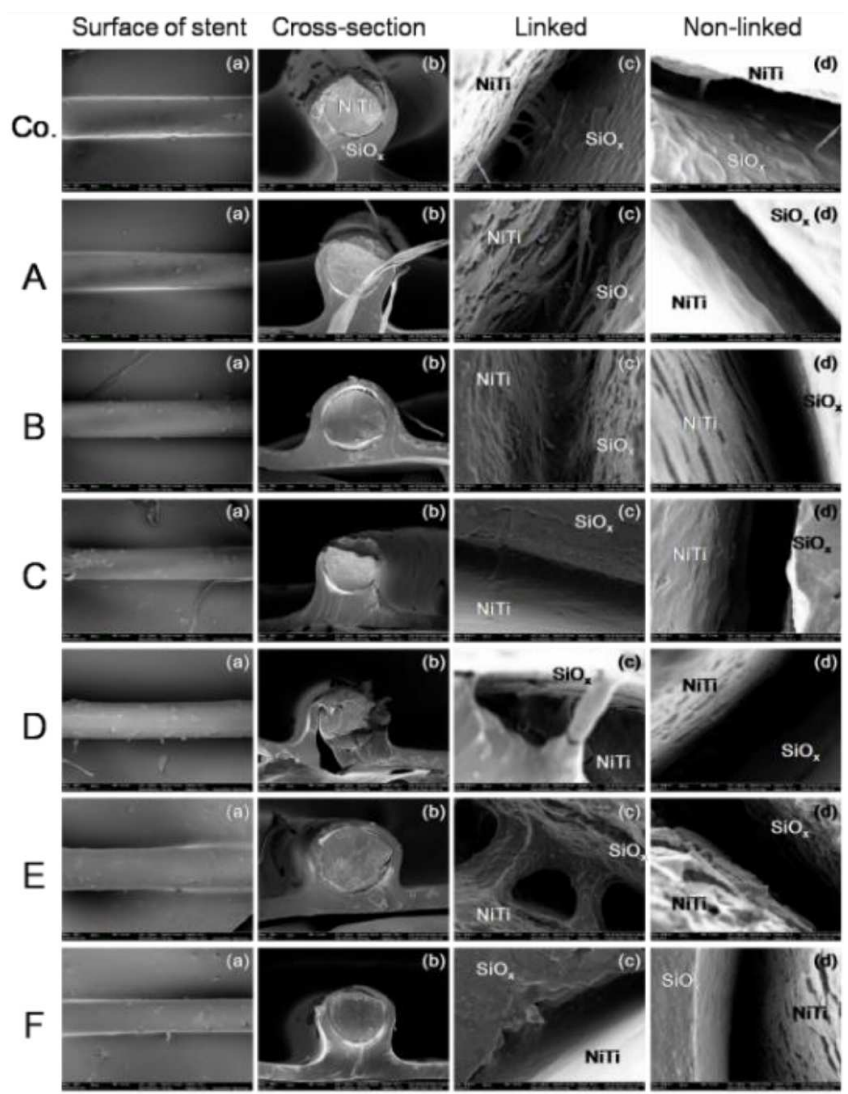

Fig. 1. Scanning electron micrographs. First column from left shows surfaces of horizontally straight parts of the stents with the silicone. The second column, vertical cross section of the straight part. The third column, strongly magnified linked parts of the cross section between the silicone and the primer-treated NiTi strip. The forth column, the non-linked parts. Index $x$ of $\mathrm{O}_{x}$ symbol means 1 or 2 , and others, which is the number of bound oxygen atoms.

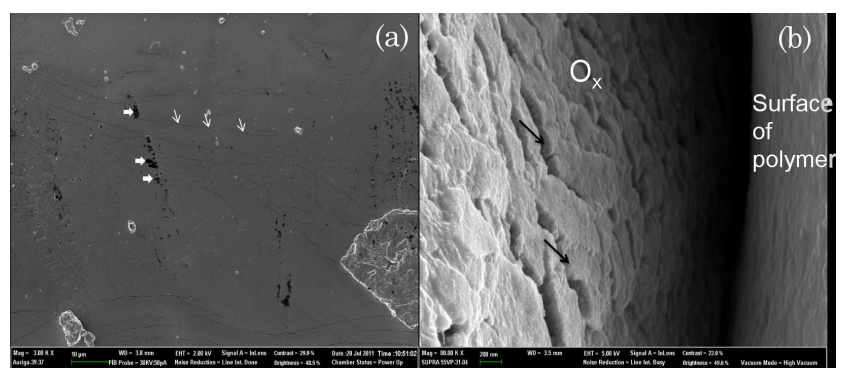

Fig. 2. Scanning electron micrographs showing: (a) the surface of silicone that has many long cleavages (small arrows) and peeling of large areas (large arrows), and (b) the oxide layer with the presence of cracks on lateral face of NiTi strip (dark arrows).

the NiTi strips are exposed to the aqueous environment of the human body [17]. This observation implies that not only thick oxide layer but also well-structured oxide layer is necessary for better corrosion-resistance.

To quantitatively evaluate the oxide layer on the NiTi strips, which prevents the exposure of Ni to the solution, anodic potentiodynamic polarization test was applied. An anodic potentiodynamic polarization test is a technique where the potential of the sample is varied at a selected rate by the application of current through the electrolyte. It is the most commonly used polarization testing method for measuring corrosion resistance. If the surface of a NiTi strip is well linked and covered with silicone or a well-structured thick oxide layer is formed on the strip, the corrosion resistance might be higher. The anodic potentiodynamic polarization behavior of the samples is depicted by the Tafel plot in Fig. 3, revealing different responses in the deaerated $0.9 \%$ sodium chloride solution. The corrosion potential $E_{\text {corr }}$ and the

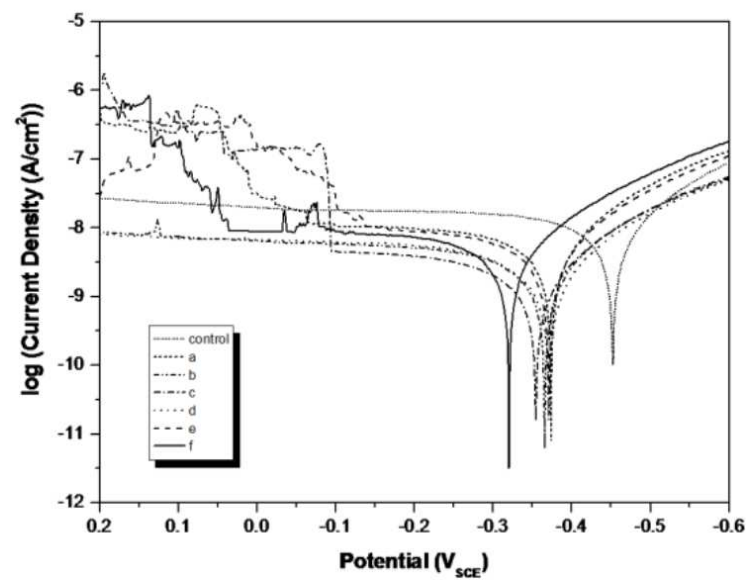

Fig. 3. Potentiodynamic polarization curves of the stents and the NiTi wires in deaerated $0.9 \%$ sodium chloride solution at room temperature, measured at a scanning rate of $5 \mathrm{mV} / \mathrm{s}$.
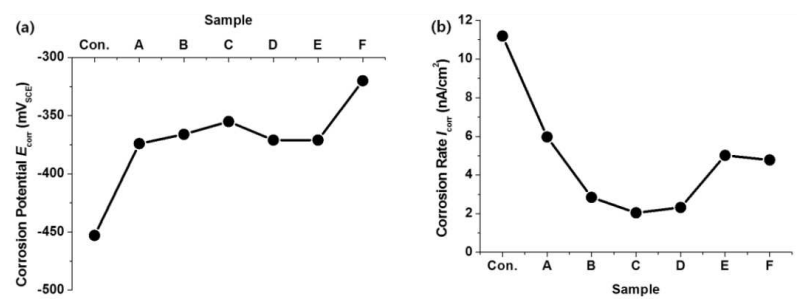

Fig. 4. Comparison of the electrochemical parameters of the NiTi alloy strips after treatment with a primer (Con) or plasma in various conditions $(\mathrm{A}-\mathrm{F})$. Corrosion potential (a) and corrosion rate (b) were determined from the polarization curves in Fig. 3. There is clear difference between the chemically treated and the physically treated strips.

corrosion rate $I_{\text {corr }}$ extracted from the curves are shown in Fig. 4. The corrosion potentials of all plasma-treated NiTi $(\mathrm{A}-\mathrm{F})$ were shifted in the noble direction compared to the control (primer-treated), and the corrosion rates of all samples were less than half of that of the control sample, indicating much higher corrosion resistance (Fig. 4). We cannot find significant differences among different plasma etching conditions with different oxygen rate, applied voltage, and treatment time. However the high oxygen flow $(\mathrm{F})$ gives highest corrosion potential 
(up to $133 \mathrm{mV}$ SCE to control), and long time plasma exposure (B and D) makes generally low corrosion rate (down to $21 \%$ of control). Although we could not find the differences in silicon adhesion on NiTi morphologically with FESEM, we have observed more than two-fold increases in corrosion potential and decrease in corrosion rate. This implies that plasma-treatment induces more uniform or thicker oxide layer on NiTi strips, which enhances corrosion resistance much more than treatment with primer. The oxide thickness of each sample can be measured using TEM analysis and their structure can be analyzed using electron energy loss spectroscopy.

\section{Conclusions}

We have induced a mechanical bond between polymer and stent substrate by using primer which is currently used clinically and by plasma treatment method, which is being tested for manufacturing purposes. The interface was characterized by FESEM and the corrosion rate was analyzed by anodic potentiodynamic polarization test. It was found that the bond at the interface was only partial in both primer and plasma-treated samples. On the contrary, the results of the potentiodynamic polarization test have shown that the corrosion rates of the plasmatreated samples were lower than the half of that of the primer-treated stents, indicating a higher corrosion resistance. This suggests that the plasma treatment makes the oxide layer more uniform or thicker, which may also facilitate the adherence of silicone to the surface. Further work will address the elucidation of the thickness and atomic structure of titanium oxide layer made by plasma treatment.

\section{Acknowledgments}

This work was supported by the National Research Foundation (NRF) of Korea Grant, funded by the Korean Government (No. 2010-0029418).

\section{References}

[1] A.R. Pelton, J. DiCello, S. Miyazaki, Min. Invas. Ther. Allied Technol. 9, 107 (2000).

[2] D. Stoeckel, A. Pelton, T. Duerig, Eur. Radiol. 14, $292(2004)$.
[3] O. Fukushima, T. Yoneyama, H. Doi, T. Hanawa, Dent. Mater. J. 25, 151 (2006).

[4] M. Es-Souni, M. Es-Souni, H. Fischer-Brandies, Anal. Bioanal. Chem. 381, 557 (2005).

[5] S.A. Shabalovskaya, Bio. Mater. Eng. 12, 69 (2002).

[6] M.M. Mazumder, S. De, S. Trigwell, N. Ali, M.K. Mazumder, J.L. Mehta, J. Bio. Sci. Polymer Edition 14, 1351 (2003).

[7] S. Trigwell, S. De, R. Sharma, M.K. Mazumder, J.L. Mehta, J. Bio. Mat. Re. Part B: App. Biomat. 76B, 241 (2006)

[8] P. Hale, S. Turgeon, P. Horny, F. Lewis, N. Brack, G. van Riessen, P. Pigram, D. Mantovani, Langmuir 24, 7897 (2008).

[9] P. Molitor, V. Barron, T. Young, Int. J. Adhes. Adhes. 21, 129 (2001).

[10] E. Dayss, G. Leps, J. Meinhardt, Surf. Coat. Tech. 116-119, 986 (1999).

[11] N. Choi, H.K. Lee, S. Jung, K.H. Park, J. Nanosci. Nanotechnol. 10, 3299 (2010).

[12] K. Navaneetha Pandiyaraj, V. Selvarajana, M. Pavese, P. Falaras, D. Tsoukleris, Current Appl. Phys. 9, 1032 (2009).

[13] M. Diefenbeck, T. Mückley, C. Schrader, J. Schmidt, S. Zankovych, J. Bossert, K.D. Jandt, M. Faucon, U. Finger, Biomater. 32, 8041 (2011).

[14] T. Kitsugi, T. Nakamura, M. Oka, Y. Senaha, T. Goto, T. Shibuya, J. Biomed. Mater. Res. 30, 261 (1996).

[15] M. Park, A. Calabrich, A.T. Maciel, Rev. Bras. Ter. Intensiva. 23, 176 (2011).

[16] K. Subramanian, J. Mickalonis, Electrochim. Acta 50, 2685 (2005).

[17] C. Trépanier, M. Tabrizian, L.H. Yahia, L. Bilodeau, D.L. Piron, J. Biomed. Mater. Res. 43, 433 (1998).

[18] S. Tomić, R. Rudolf, M. Brunčko, I. Anžel, V. Savić, M. Colić, Eur. Cell. Mater. 29, 58 (2012).

[19] A. Fossati, M. Di Ferdinando, A. Lavacchi, A. Scrivani, C. Giolli, U. Bardi, Coatings. 1, 3 (2011). 